\title{
Structural intensity analysis of flat plates based on digital
} stroboscopic holography measurements

\author{
F. Pires ${ }^{\text {a,b, }}{ }^{*}$, P. G. G. Muyshondt ${ }^{a}$, W. Keustermans ${ }^{a}$, \\ S. Vanlanduit ${ }^{a, b}$, N. B. Roozenc, J. J. J. Dirckx ${ }^{a}$ \\ a University of Antwerp, Laboratory of Biophysics and Biomedical Physics, \\ Department of Physics, Groenenborgerlaan 171, 2020 Antwerp, Belgium. \\ ${ }^{\mathrm{b}}$ Vrije Universiteit Brussel, Department of Mechanical Engineering, \\ Pleinlaan 2, 1050 Brussels, Belgium. \\ ${ }^{\mathrm{C}}$ Katholieke Universiteit Leuven, Laboratory of Acoustics, Soft Matter and Biophysics,
Department of Physics and Astronomy, Celestijnenlaan 200D 3001 Leuven, Belgium.
}

\begin{abstract}
The analysis of structural intensity vector fields has shown to be a practical approach to characterize the energy flow in plate-like structures and to localize regions of injected or absorbed power. Such an analysis is performed by differentiating measured velocity or displacement fields of a sample. The quality of such a study strongly depends on the spatial resolution of the deformation data and its signal-to-noise ratio. The digital stroboscopic holography concept is presented in this work and used as a tool to record such deformations on a flat plate, which is in direct contact with a shaker and a damper. The current set-up could provide the recording of displacement fields at a high spatial resolution showing little corruption by noise. These conditions permitted the measurement of deformation patterns containing short wavelengths, which were later used as inputs for the structural intensity analysis. By calculating the spatial derivatives of several out-of-plane displacement fields, the energy flowing through the plate was estimated and the position of the external devices (shaker and damper) could be identified by locating regions of the energy dispersion or convergence. The location of these specific regions was made even clearer by calculating the divergence of the energy flow.
\end{abstract}

Keywords: digital stroboscopic holography, source and sink localization, spatial Fourier transform, structural intensity, energy transmission

PACS: 46.70.-p, 46.70.De, 42.40.-i , 47.80.Jk

*Corresponding author:

E-mail: felipe.pires@uantwerpen.be

Address: Groenenborgerlaan 171, G.U.341 - 2020 Antwerpen, Belgium 


\section{Introduction}

Structural intensity (SI) is a concept that describes the energy being transported by elastic waves in a structure. A commonly studied case is the energy flowing through thin plates. If the necessary simplifications are assumed, it is possible to assess the transmission of energy using the measurements of the sample's out-of-plane motion. In the past, several attempts have been made to reach this goal using contact method approaches, such as accelerometers [1,2]. The main limitation of such an approach is that it alters the system's behavior due to the added mass of the transducer. Later, non-contact methods which do not add mass to the measured structure were used to detect surface motions, i.e., the Laser Doppler Vibrometry (LDV) [3-6], acoustic-holography [7], electronic speckle interferometry [8] or holographic interferometry [9]. These techniques are not only non-contact but also allow the measured displacement or velocity fields to be recorded with higher spatial resolution than the mentioned pioneering studies. Moreover, the grid sizes from non-contact measurements found in literature vary from $165(15 \times 11)$ [10] to $15876(126 \times 126)$ [8] measurement points.

In turn, the spatial derivatives of the out-of-plane displacement fields provide the necessary inputs for the assessment of SI and the localization of sources and sinks. Many studies have already been carried out to estimate the energy flow in the form of vector fields or by calculating its divergence, which allows clear visualization of vector's dispersion or convergence $[4,5,11-15]$.

The Digital Stroboscopic Holography (DSH) is presented in this paper as another alternative among the available non-contact methods to study the structural intensity. A set-up that measures the out-of-plane displacement of a harmonically excited thin flat plate by using this technique is presented. After calibration, a total of 305.373 measurement points (411 x 743) were achieved and the images were processed for further analysis.

\section{Theory}

\subsection{Power and structural intensity}

The expressions regarding the definition of power [3,15-18] and structural intensity in plates [12-14,19-21] are well documented in literature and are therefore just briefly reviewed here. The mean power is defined as the time-averaged product of generalized forces and related in-phase velocities. In the particular case where it is desired to investigate the transferred power injected in the structure at an excitation point and by assuming that it behaves harmonically, the mean transmitted power can be directly calculated by

$$
P=-\frac{1}{2} \operatorname{Re}\left\{\tilde{F} \cdot \tilde{v}^{*}\right\}
$$

where $\tilde{F}$ is the force with which the structure is excited in the normal direction, $\tilde{v}$ is the out-ofplane velocity at the point of excitation, $\sim$ denotes the complex representation of the mentioned field and $*$ denotes the complex conjugate operator. Depending on the value which is extracted from Eq. (1), it can be interpreted if the power is either injected or absorbed at the 
excitation point. In the particular case where power is injected into the system, the absolute phase difference between the force $\tilde{F}$ and the velocity $\tilde{v}$ is less than $\frac{\pi}{2}$ radians. The smaller the phase's difference between these 2 quantities, the greater the injected power.

On the other hand, if the absolute relative phase is greater than $\frac{\pi}{2}$ radians, power is absorbed from the system. In cases where the phase difference of the force and velocity is nearly $\pi$ radians, the measured point is considered to have strong viscous damping behavior. Furthermore, if $\tilde{F}$ and $\tilde{v}$ are totally out-of-phase, i.e. with a phase difference equal to $\frac{\pi}{2}$ radians, no power is transmitted, which means that the measured point behaves as a combination of a mass and spring.

In flat plates, the power transmission is caused by the interaction between internal generalized forces and velocities. One way to assess the energy flow within the plate is by assuming that the sample behaves as a Kirchhoff plate. Since the flexural waves are the wave types which most contribute to the vibration energy in plates $[9,16]$, Eq.(1) can be simplified and recast in terms of

$$
\begin{gathered}
\mathbf{I}=\left(I_{x}, I_{y}\right), \\
I_{x}=-(\pi f) \operatorname{Im}\left\{\tilde{Q}_{x} \cdot \widetilde{w}^{*}+\widetilde{M}_{x y} \cdot \frac{\partial \widetilde{w}^{*}}{\partial y}+\widetilde{M}_{x} \cdot \frac{\partial \widetilde{w}^{*}}{\partial x}\right\}, \\
I_{y}=-(\pi f) \operatorname{Im}\left\{\tilde{Q}_{y} \cdot \widetilde{w}^{*}+\widetilde{M}_{x y} \cdot \frac{\partial \widetilde{w}^{*}}{\partial x}+\widetilde{M}_{y} \cdot \frac{\partial \widetilde{w}^{*}}{\partial y}\right\},
\end{gathered}
$$

where $\widetilde{Q}_{x}$ and $\widetilde{Q}_{y}$ denote out-of-plane shear forces, $\widetilde{M}_{x}$ and $\widetilde{M}_{y}$ the bending moments, $\widetilde{M}_{x y}$ the twisting moment, $f$ the excitation frequency, $\widetilde{w}$ the out-of-plane displacement field and $\mathbf{I}$ the active SI vector field per unit length $(\mathrm{W} / \mathrm{m})$.

The right-hand terms of Eq. (3) and Eq. (4) depend solely on the material properties and on the out-of-plane displacement field $\widetilde{w}$ of the plate. If the system is considered to behave in accordance with the Kirchhoff's theory of thin plates, the shear forces and bending moments can be estimated as functions of the out-of-plane displacements:

$$
\begin{gathered}
\widetilde{Q}_{x}=-\widetilde{D}\left[\frac{\partial^{3} \widetilde{w}}{\partial x^{3}}+\frac{\partial^{3} \widetilde{w}}{\partial x \partial y^{2}}\right], \\
\widetilde{Q}_{y}=-\widetilde{D}\left[\frac{\partial^{3} \widetilde{w}}{\partial y^{3}}+\frac{\partial^{3} \widetilde{w}}{\partial x^{2} \partial y}\right], \\
\widetilde{M}_{x}=\widetilde{D}\left[\frac{\partial^{2} \widetilde{w}}{\partial x^{2}}+v \frac{\partial^{2} \widetilde{w}}{\partial y^{2}}\right], \\
\widetilde{M}_{y}=\widetilde{D}\left[\frac{\partial^{2} \widetilde{w}}{\partial y^{2}}+v \frac{\partial^{2} \widetilde{w}}{\partial x^{2}}\right]
\end{gathered}
$$




$$
\begin{aligned}
\widetilde{M}_{x y} & =\widetilde{D}(1-v) \frac{\partial^{2} \widetilde{w}}{\partial x \partial y} \\
\widetilde{D} & =\frac{E(1+i \eta)}{\left(1-v^{2}\right)} \frac{h^{3}}{12}
\end{aligned}
$$

where $E$ is the plate's Young's modulus; $h$ is the thickness of the plate, $v$ is the Poisson's ratio and $i$ is the imaginary unit. The internal dissipation of energy is also taken into account in this study [22-24] by introducing the structural loss factor $\eta$ in Eq.(10).

If the sample contains regions where power is injected or absorbed, the SI vector fields diverge or converge at these respective locations. These phenomena become apparent by visualizing the divergence of the SI (DSI), offering the out-of-plane surface density of injected or absorbed power per unit area $\left(\mathrm{W} / \mathrm{m}^{2}\right)$,

$$
\nabla \cdot \mathbf{I}=\frac{\partial I_{x}}{\partial x}+\frac{\partial I_{y}}{\partial y}
$$

The concentrated positive values that this field provides are referred to as "sources", while the negative ones are referred to as "sinks". At this point, it becomes evident from Eqs. (5-9, 11) that the extraction of DSI maps requires the calculation of fourth-order spatial derivatives.

It must be noted that the equations related to the SI (Eqs.(2-11)) are only valid for plates whose thickness and stiffness are constant throughout its domain. If the sample contains regions where the mentioned parameters are discontinuous, the equations are not applicable at these very regions.

\subsection{Spatial derivative calculation and filtering}

Apart from the material properties and thickness of the plate, it can be noted that the calculation of Eqs. $(3,4,11)$ are solely dependent on the out-of-plane displacement and its spatial derivatives. One available method to calculate these derivatives is the image processing in the wavenumber domain by applying the Spatial Fourier Transform (SFT),

$$
\widetilde{W}\left(k_{x}, k_{y}\right)=\mathcal{F}\{\widetilde{w}(x, y)\},
$$

where $\left(k_{x}, k_{y}\right)$ denote the coordinates in the wavenumber domain, $(x, y)$ the coordinates in the spatial domain and $\mathcal{F}$ a two-dimensional SFT.

It is known that wraparound error affects the image in the $k$-space due to the discrete representation [19] of the displacement field. The influence of wraparound error on samples whose edges are clamped can be reduced by applying the zero padding technique $[19,25]$, which contours the field with bands of zeros. In the present case, this technique triples the size of the matrix on both directions after its application. 
When it is desired to extract DSI fields (Eq.(11)), spatial derivatives up to the fourth order in both directions need to be computed. The derivative operation in the wavenumber domain amplifies the contribution of high wavenumbers, which are related to noise on experimental data. Therefore, the image needs proper filtering prior to the spatial derivation, so the noise will not mask the data of interest.

A low-pass filter is applied to the data in the wavenumber domain to attenuate the amplification of measurement noise. The combination of this spatial derivation with filtering was shown to be less susceptible to corrupted data, when compared to its finite-difference concept counterpart [3-5,8,26,27]. The low-pass filter developed by Li et al. [28] is used in the present work. The filter's shape is controlled by a cut-off wavenumber, $k_{c}$, and a parameter, $\mathrm{s}$, that controls the slope variation of this window function at the cut-off wavenumber. The described equation is

$$
f\left(k_{x}, k_{y}\right)=\left\{\begin{array}{rr}
1, & \text { for } k_{r}=0, \\
1-0,5 \exp \left[-\frac{\left(\frac{k_{c}}{k_{r}}-1\right)}{s}\right] & \text { for } k_{r} \leq k_{c}, \\
0,5 \exp \left[-\frac{\left(\frac{k_{r}}{k_{c}}-1\right)}{s}\right] & \text { for } k_{r}>k_{c},
\end{array}\right.
$$

where $k_{r}$ represents the radius of the filter on the $k$-space coordinates, i.e., $k_{r}=\sqrt{k_{x}^{2}+k_{y}^{2}}$.

The low-pass filter is applied to the zero-padded displacement fields each time a first order spatial derivative is carried out [29]. Its mathematical description is

$$
\frac{\partial^{m+n}}{\partial x^{m} \partial y^{n}} \widetilde{w}(x, y)=\mathcal{F}^{-1}\left\{\left(\mathrm{i} k_{x}\right)^{m}\left(\mathrm{i} k_{y}\right)^{n} \widetilde{W}\left(k_{x}, k_{y}\right) \cdot f\left(k_{x}, k_{y}\right)^{m+n}\right\}
$$

All necessary spatial derivatives provided by a single displacement field are calculated with one combination of adjusted $k_{c}$ and $s$ parameters from the filter of Eq.(13).

\subsection{Digital stroboscopic holography}

In holography, a laser beam is split into two parts, and each of these beams is expanded using lenses. By using a combination of mirrors, one beam, called the reference beam, is directed onto the recording medium. The other beam, called the object beam, is cast onto the surface of the object. The object diffusely reflects the light towards the recording medium, where it interferes with the reference beam, thus forming a holographic interference pattern. In digital holography [30], the recording medium is a CCD device. The CCD output is read into a computer, and several methods exist to digitally reconstruct the object's light field. In the present study, we use the Fresnel approximation method [30]. After the reconstruction step, two matrices are obtained, one containing the amplitude distribution of the object light, and 
one containing its phase distribution. The amplitude distribution can be used to show an image of the object, but is of no further use. The phase distribution, however, contains the information needed to perform interferometric metrology. When a first hologram is recorded with the object in its rest state, and a second hologram is recorded with the object in a deformed position, the difference between the two phase matrices delivers the displacement of the object, scaled in radians and modulo $\frac{\pi}{2}$. To obtain deformation in units of length, a simple calibration step is needed, as the wavelength of the laser light is exactly known and corresponds to a phase change of the light of exactly $2 \pi$ [31]. In practice, some complications need to be taken into account as deformations will be obtained along the so-called sensitivity vector, the bisector between the illumination and observation direction. It is beyond the scope of the current paper to go into these methodological details, but sets of mirrors and beam combiners were used to obtain correctly calibrated measurements of the object's out-of-plane motion.

In DSH [32], short laser pulses are used to illuminate a vibrating object at specific instances of the vibration period. If the pulses are short enough, deformations maps are again obtained. Each holographic phase distribution can be subtracted from the phase distribution obtained on the object in its rest state, delivering displacement maps of the object at a certain instance of the vibration period. Alternatively, if the object's behavior is harmonic, a number of " $n$ " phase distribution maps $w\left(x, y, t_{n}\right)$ can be recorded at evenly spaced time steps within the vibration period. The set of these maps can then be transformed from the time domain to the frequency domain as follows:

$$
\widetilde{w}(x, y)=\mathcal{F}_{\mathrm{TFT}}\left\{w\left(x, y, t_{n}\right)\right\}
$$

where $\widetilde{w}(x, y)$ is the complex displacement field, and $\mathcal{F}_{\text {TFT }}$ denotes the temporal Fourier Transform. When $\widetilde{w}(x, y)$ is calculated, it can be processed by using Eq.(12) and Eq.(14) for the purpose of analyzing power transmission.

\section{Materials}

In the present work, an $18 \mathrm{~cm} \times 13 \mathrm{~cm} \times 0,5 \mathrm{~mm}$ flat aluminum plate with firmly clamped edges was used as a demonstration sample. It was assumed that the Poisson's coefficient and the Young's modulus of the plate were 0,3 and $69 \mathrm{GPa}$; respectively. The internal damping loss factor $\eta$ of the sample was estimated on the basis of the so-called poly-reference modal parameter estimation algorithm [33]. By assessing a system's frequency response function, the poles of the system's eigenfrequencies are used to extract the damping loss factor [34] with

$$
\eta_{k}=-2 \frac{\operatorname{Re}\left\{\lambda_{k}\right\}}{\left|\lambda_{k}\right|}=2 \xi
$$

being $k$ the subscript of the identified mode, $\lambda$ the stabilized pole extracted from the FRF and $\xi$ the damping ratio. The loss factor was estimated on the basis of the sample's first eigenmode, which provided values around $\eta_{1}=0,02$. It was assumed that the plate could be represented by a constant loss factor throughout the frequency and an internal damping of $2 \%$ was used in Eq.(10). 
To identify concentrated sources on the plate, a shaker was used to harmonically excite the structure on a specific point. The tip of that shaker was directly attached to the plate and had a diameter of $2 \mathrm{~mm}$ at the point of attachment. To absorb power, a piece of synthetic viscoelastic urethane polymer (Thorlabs, SB12B Sorbothane Sheet, NJ, USA) was connected to the plate at a different location. The attachment of this damper with the plate had a circular area whose diameter was approximately $4 \mathrm{~mm}$. These devices and the plate are illustrated in Figure 1.

The polymer behaves as a viscous damper depending on which frequency the sample is excited. To estimate the frequency ranges at which the dissipation of vibrational energy takes place, a load cell (PCB Piezotronics, 208C01 SN LW39846, Depew, NY, USA) and a LDV system (Polytec, OFV-5000 controller and OFV 353 - sensor head, Waldbronn, Germany) were incorporated in the setup. The signals from both devices were read out on an oscilloscope (Tektronix, AFG 3022C, Beaverton, OR, USA) and the stimulation signals were delivered to the shaker by a function generator (Tektronix, AFG 3022C, Beaverton, OR, USA).

The DSH setup made use of a continuous-wave frequency-doubled YAG laser (CNI, MSL-FN$532-200 \mathrm{~mW}$, Changchung, China) delivering a $200 \mathrm{~mW}$ green laser beam with a wavelength of $532 \mathrm{~nm}$. The stroboscopic pulses were obtained by chopping the laser beam using an optoacoustic modulator (Isomet 1205C-2, Springfield, VA, USA) and a duty cycle of $2 \%$ of the vibration period. For the present paper, 16 time steps were used in each vibration period to improve the signal to noise ratio. With these settings and after calibration, Eq.(15) was used to convert the displacement fields from the time domain to the frequency domain, delivering matrices with a total of 305.373 measuring points: 411 rows and 743 columns.

\section{Methods}

The purpose of this work is to assess the $\mathrm{SI}$ and their respective DSI fields of the harmonically excited plate. To do so, preliminary studies were done to identify at which excitation frequencies the displacement fields should be recorded with the DSH set-up.

The identification of sinks is not trivial since the damping provided by the polymer depends on the excitation frequency and the position of the damper. When the damping material is located on a region whose out-of-plane displacement is negligible apart from the clamped edges or when it acts as combination of spring and mass, no sink will be visible in the DSI fields. Due to this phenomenon, it is necessary to first identify at which frequencies the available damping material would strongly absorb power, i.e., behave as a viscous damper.

As it was explained in Section 2.1, if there is strong power absorption from the damper, the phase difference between the force and velocity is nearly $\pi$ radians. This interpretation of the power's definition (Eq.(1)) was used to search for frequencies on which the mentioned polymer strongly acted as a damper. Therefore, the set-up (Figure 1) was built in such a way that the damper's force and velocity could be estimated with the oscilloscope while the excitation frequency was provided by the generator.

One side of the viscoelastic urethane polymer was connected to the plate while the other was attached to the fixed load cell. A reflective tape was attached at the same location on the 
opposite side of the plate to record its velocity with LDV. The force and velocity measurements were both visualized on the oscilloscope and the generator provided the power to the shaker.

It is worth noting that the measured force at one end of the damper is not equal to the force applied to its attachment to the plate due to internal dynamic effects in the damper. This approach was just used to support the search for excitation frequency ranges in which a strong absorption could be found.

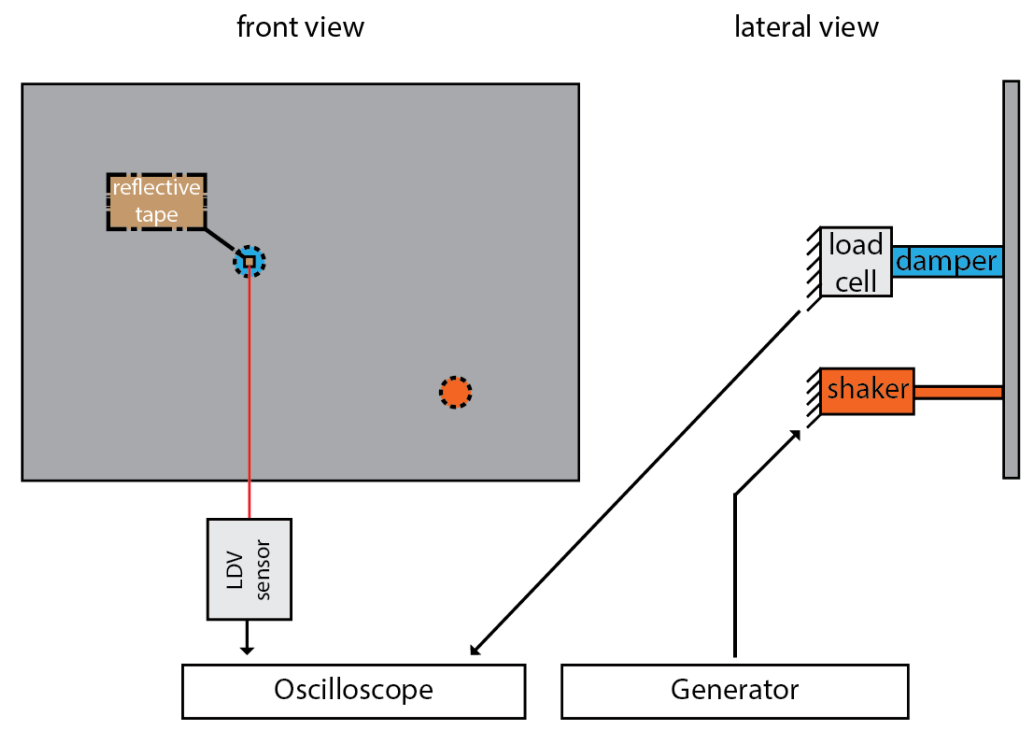

Figure 1 - Set-up configuration

The excitation frequency delivered to the plate was manually changed on the generator while the measured force and velocity signals at the damped point were inspected on the oscilloscope. The excitation frequencies for which the phase difference between the force and the velocity is close to $\pi$ radians were searched for in this procedure. These specific frequencies were then selected as inputs to excite the plate for measurements with the DSH set-up. The resulting displacement fields were processed with Eq.(14) to determine the spatial derivatives and thus compute the energy paths and their respective DSI fields by using Eqs.(2-4,11). After this computation, it was verified whether their source's and sink's locations matched the position of the shaker and the damper.

Up to this part, it is evident that the external devices (a shaker and a damper) change the thickness and stiffness of the plate at the regions where they are attached. As mentioned in Section 2.1, the Eqs.(2-11) will not hold for these very regions, since the parameters $h$ and $E$ are assumed to be constant throughout the plate's surface. To test the validity of these equations for the sample containing these heterogeneities, a model which is similar to the presented set-up was created and its SI was analyzed. This model and its corresponding energy flow case are shown in the Appendix and have shown that the added components add little inconsistencies to the SI study due to their small sizes. 


\section{Results}

The excitation frequencies for which the force and velocity measurements had phase differences near $\pi$ radians were searched for using the oscilloscope and 6 frequencies indicated that the damper was absorbing power: 1154, 1349, 1614, 2000, 2277 and $2532 \mathrm{~Hz}$. Afterwards, the DSH measurements were made while the plate was being excited at these very frequencies. The magnitude and phase of the generated displacement maps can be seen in Figure 2 .

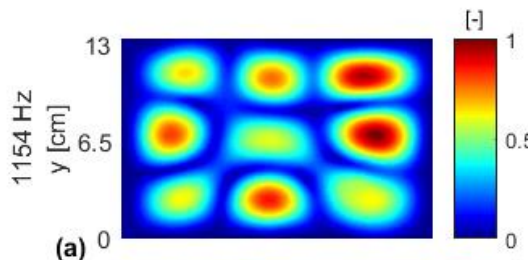

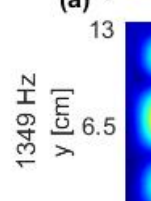

(c)

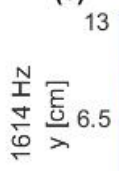

(e)
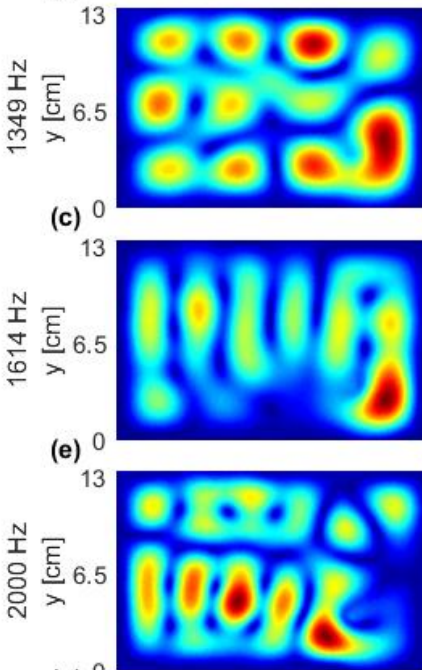

(g)

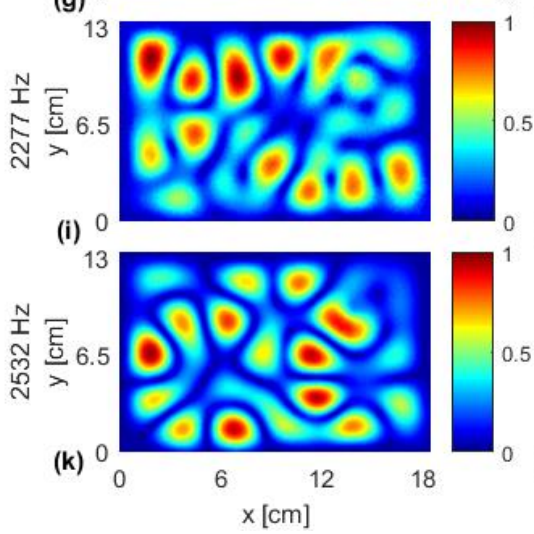

(b)
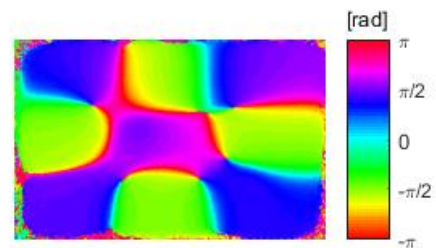

(d)
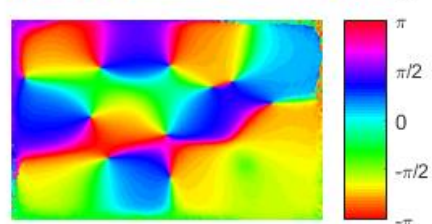

(f)
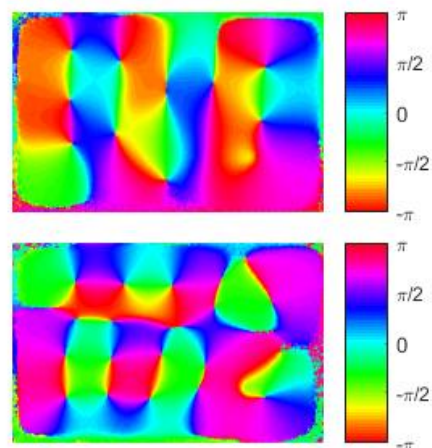

(h)
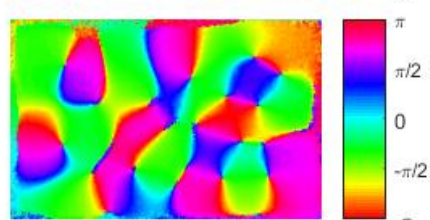

(j)

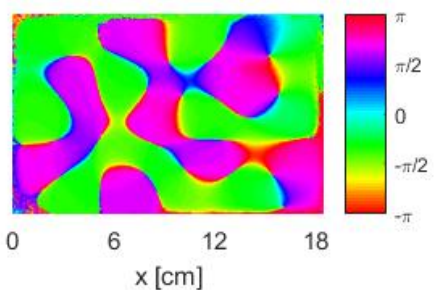

Figure 2 - Magnitude and phase maps of displacement fields. The first column presents the displacement magnitudes normalized by their respective maximum values [-] while the second column shows the relative phase [rad]. The displacement fields were generated at $1154 \mathrm{~Hz}$ [(a)-(b)], $1349 \mathrm{~Hz}$ [(c)-(d)], $1614 \mathrm{~Hz}$ [(e)-(f)], $2000 \mathrm{~Hz}$ [(g)-(h)], $2277 \mathrm{~Hz}$ [(i)-(j)] and $2532 \mathrm{~Hz}[(\mathbf{k})-(\mathrm{I})]$.

After recording the displacement maps, zero padding was applied and their respective wavenumber domain fields were acquired through Eq. (12). The latter are displayed in the first column of Figure 3 and correspond to the SFT of the displacement fields (Figure 2). The wavelength's most important information are concentrated at $115 \mathrm{rad} / \mathrm{m}$ for the excitation 
frequency of $1154 \mathrm{~Hz}$ and increases up to $180 \mathrm{rad} / \mathrm{m}$ for the highest displayed frequency (2532 $\mathrm{Hz})$.

The next step concerns the spatial differentiation of these images in the wavenumber domain while selecting a proper cut-off wavenumber $k_{c}$ that preserves the most relevant displacement's wavelengths while discarding the noise at higher wavenumbers. The chosen $k_{c}$ and $s$ that could provide recognizable SI and DSI maps for all displacement fields were 315 $\mathrm{rad} / \mathrm{m}$ and 0,6; respectively. In the first column of Figure 3 the filter's cut-off wavenumber, $k_{c}$, is also displayed in the wavenumber fields as a white circle.

In the second column of Figure 3 the SI vector fields are shown for all the recorded frequencies. The paths in which the energy flows become more complex with the increase of frequency. The visualization of the sources and sinks, i.e., the regions where the $\mathrm{SI}$ are diverging or converging, is made possible by applying the divergence operator on the SI vector fields, (Eq.(11)) as shown in the third column of Figure 3. Even though the SI vector fields at different frequencies are distinguishable, their divergence fields look similar, i.e., with concentrated negative and positive values at regions where external forces are applied, and values near zero on the free-zones. 


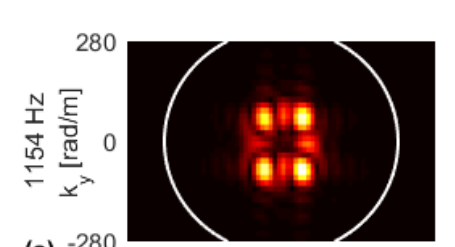

(a)

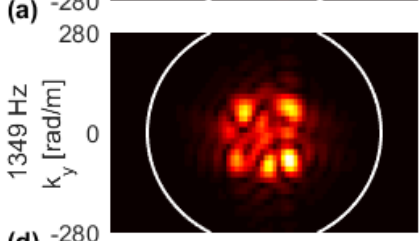

(d) -280

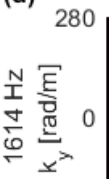

(g) -28
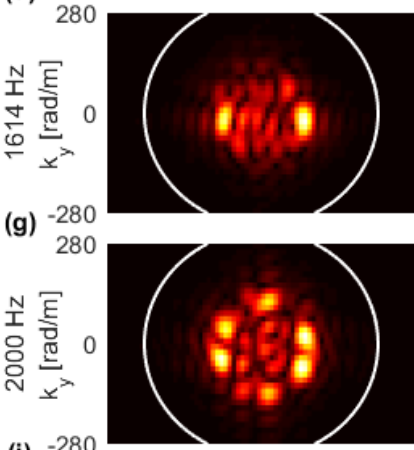

(j) -280

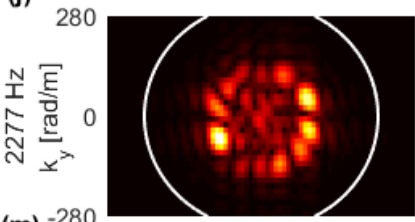

(m) -280

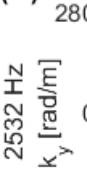

(p) -280

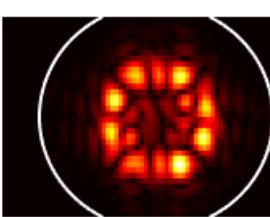

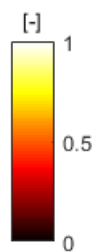
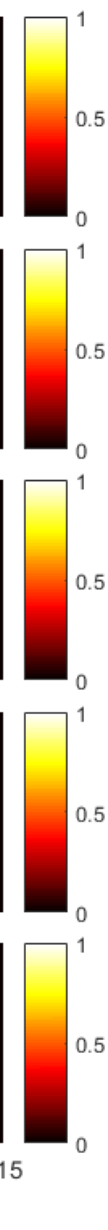

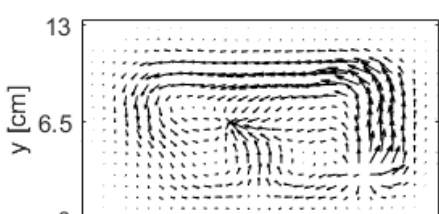

(b)

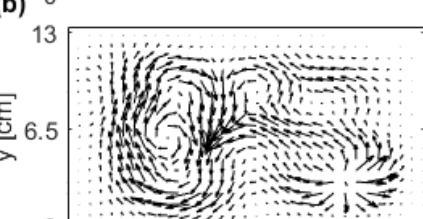

(e)

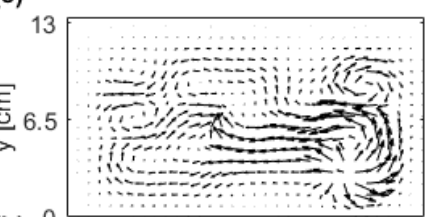

(h)

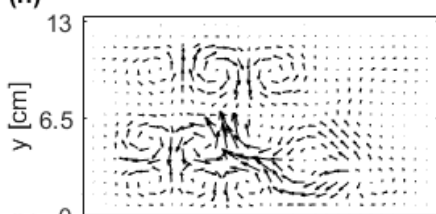

(k)

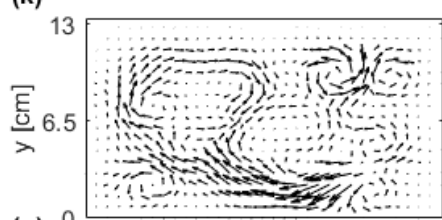

(n)

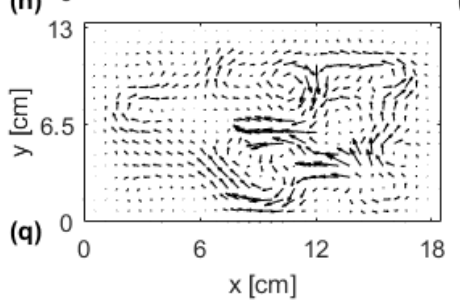

(c)

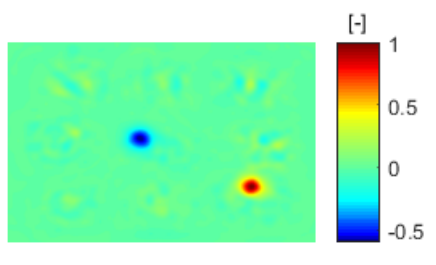

(f)

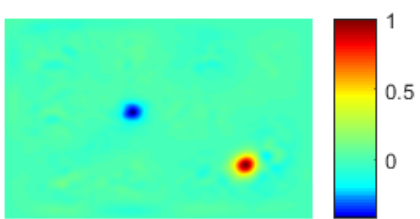

(i)

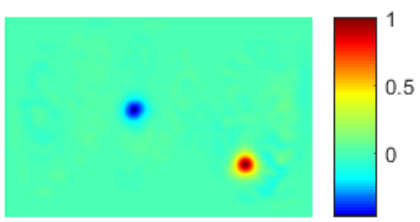

(I)

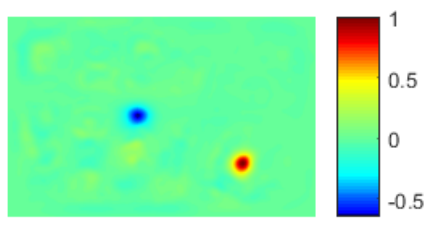

(o)

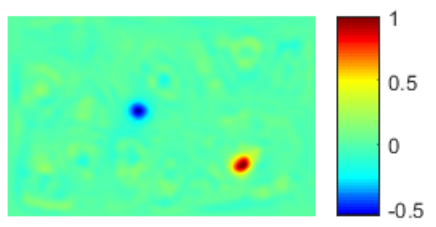

(r)

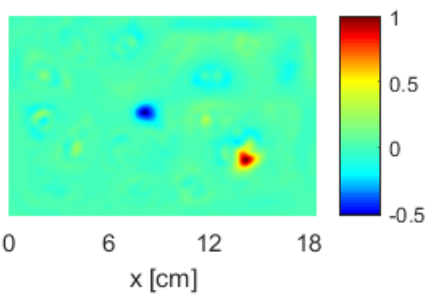

Figure 3 - Wavenumber domain representation of the displacement fields [(a)-(d)-(g)-(j)-(m)-(p)], averaged SI vector fields [(b)-(e)-(h)-(k)-(n)-(q)] and divergence of structural intensity [(c)-(f)-(i)-(l)-(o)-(r)]. The wavenumber fields are normalized with respect to their own maximum value [-] and the divergence of structural intensity fields are also normalized to their respective maximum values [-]. The value of $k_{c}$ is $315 \mathrm{rad} / \mathrm{m}$ and is depicted as a white circles on the first column.

\section{Discussion and Conclusions}

The displacement fields from a clamped flat plate which was connected to a shaker and a damper were processed to assess the SI and their respective DSI fields. The frequency ranges at which the installed damper approximately resembled a viscous load were identified by monitoring the force and velocity at the region where the damper was mounted. With this approach, 6 excitation frequencies were selected to excite the sample and to record the out-ofplane displacements between 1 and $3 \mathrm{kHz}$ with a high spatial resolution (a total of 305.373 measurement points). 
It was shown that most relevant wavelengths of the recorded displacement fields are located between $115 \mathrm{rad} / \mathrm{m}$ and $180 \mathrm{rad} / \mathrm{m}$ (first column of Figure 3). It is clear that the recording of these patterns demand a high spatial resolution, which was successfully captured with the DSH set-up. To our knowledge, this is the first study using displacement patterns with such a short wavelengths for the purpose of extracting SI information.

Moreover, the presented image processing results provided the vibrational energy paths travelling in the plate at all selected frequencies (second column of Figure 3 ). By examining the SI vector fields, it is possible to locate the regions where the vectors are converging or diverging and that they correspond to the damper's and shaker's position. The visualization of the areas where these external forces are being applied becomes even clearer by calculating the divergence of the SI (third column of Figure 3).

These results have shown that the DSH concept was able to provide displacement fields from which a SI study could be made. This concept in combination with the current algorithm to estimate spatial derivatives were able to present meaningful information with respect to the energy paths and the location of power injection or absorption.

\section{Acknowledgements}

Financial support for this work was supplied by the Research Foundation of Flanders (FWO), (grant No. G049414N) and also carried out in the framework of H2020-MSCA-RISE-2015 (acronym PAPABUILD, proposal number 690970). The authors would also like to acknowledge the support given by OPTIMESS (Optical Measurement Techniques for Structures \& Systems). 


\section{Appendix}

A model resembling the set-up presented in Figure 1 has been developed in a FiniteElement-Method software (COMSOL ${ }^{\circledR}$ Multiphysics 5.2 a, Burlington, Massachusetts, USA). The dimensions of the plate, shaker's tip and damper were measured from the set-up to create the geometry's components (Figure A.1), being the plate modeled as a shell and the remaining components as solids. The material properties of each component of this model are presented in Table A.1. The plate was clamped at its edges, a fixed constraint was applied to the damper's surface opposite to its attachment to the plate and a harmonic motion at $100 \mathrm{~Hz}$ was set on the shaker's tip at the direction normal to the plate's surface.

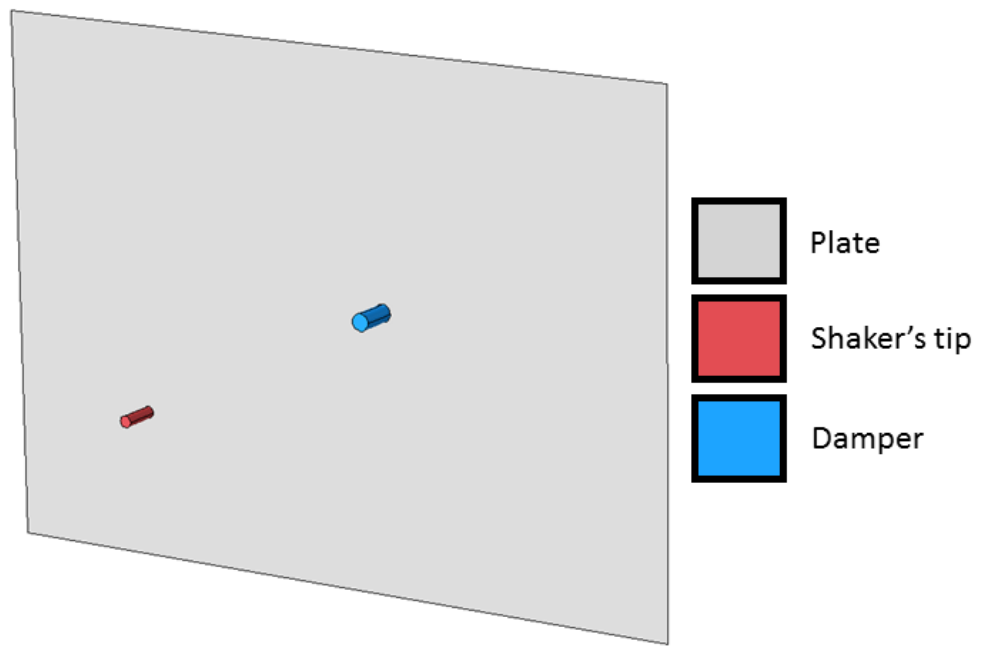

Figure A.1 - Model resembling the experimental set-up

\begin{tabular}{|c|c|c|c|}
\hline & Plate & Shaker's tip & Damper \\
\hline $\boldsymbol{E}(\mathrm{GPa})$ & 69 & 100 & $1,7 \mathrm{e}^{-3}$ \\
\hline $\boldsymbol{\rho}\left(\mathrm{kg} / \mathrm{m}^{3}\right)$ & 2.700 & 7.400 & 130.000 \\
\hline $\boldsymbol{\eta}(-)$ & 0,02 & - & 0,5 \\
\hline
\end{tabular}

Table A.1 - Material properties of the components in the model

The FEM model directly provides the generalized forces and displacement fields after computation $\left(\tilde{Q}_{x}, \tilde{Q}_{y}, \widetilde{M}_{x}, \widetilde{M}_{y}, \widetilde{M}_{x y}\right.$ and $\left.\widetilde{w}\right)$ and can be directly substituted in Eqs.(3,4), so the SI can be visualized. However, as mentioned in Section 2.1, these equations are only valid on the regions where the plate is not connected to the external devices. Figure A.2 presents the computed $\mathrm{SI}$ of the model and which also omits the energy paths at the heterogeneity regions (shaker's tip and damper). 


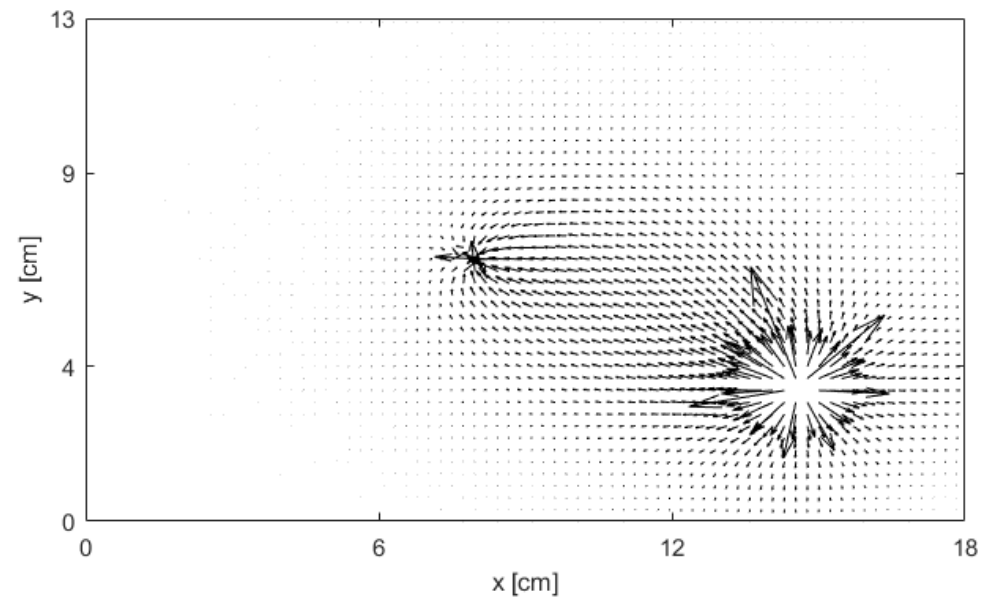

Figure A.2 - SI vector field extracted from the model. The vectors are absent on the regions were the shaker and damper were coupled to the plate, since the SI equations are not applicable there.

After visualizing the energy path on the plate's free-zones directly from the model, the SI that is extracted on the basis of the presented algorithm was used for further comparison. It is worth noting that the method to differentiate the displacement fields (Section 2.1 and 2.2) displays the $\mathrm{SI}$ on the plate's whole domain, even at the regions where the external devices were installed. The Eqs.(2-11) were implemented as if the plate was homogeneous with respect to the thickness and stiffness, i.e., $E=69 \mathrm{GPa}$ and $h=5 \mathrm{~mm}$. The displacement fields' derivatives were carried out with a cut-off wavenumber equal to the ones used on the experimental data $\left(k_{c}=315 \mathrm{rad} / \mathrm{m}\right)$ and the energy paths were estimated (Figure A.3).

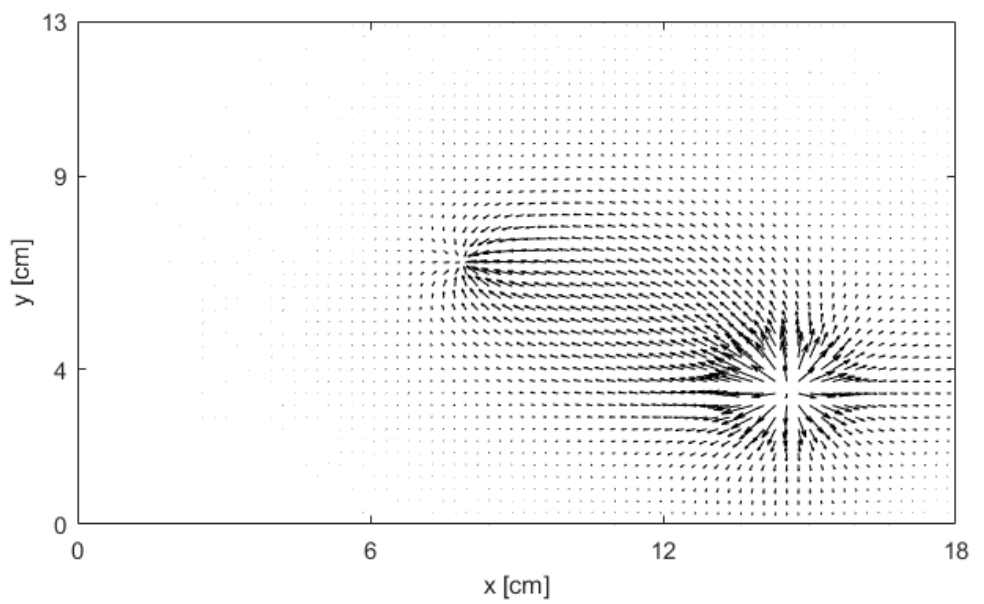

Figure A.3 - SI vector field assessed through the spatial derivatives of the synthetic out-of-plane displacement. This result was obtained by assuming that the plate was homogeneous and the influence of the attached devices were neglected.

If the energy paths of both figures are compared, it can be seen that SI vector fields on the free-zones suffered almost no changes in terms of the vector fields' directions and magnitudes. This is the case, since the plate's material properties and thickness are correctly described in the mentioned region. However, the vector fields which are the closest to the shaker's tip and damper in Figure A.3 were the most affected by the proposed algorithm. This issue is caused partially by the incorrect description of the thickness and stiffness at these very regions. 
Moreover, the choice of the cut-off wavenumber also influences on the smearing of these fields, since the choice of lower cut-off wavenumbers results in smeared SI and DSI.

Despite the limitation imposed by shaker and damper on the proposed algorithm, the $\mathrm{SI}$ is still accurately displayed on the free-zones and the location of where the vectors spread out or converge is also preserved. By taking the divergence of the SI fields presented in Figure A.3, the location of the power's source and sink becomes more evident and is presented in Figure A.4.

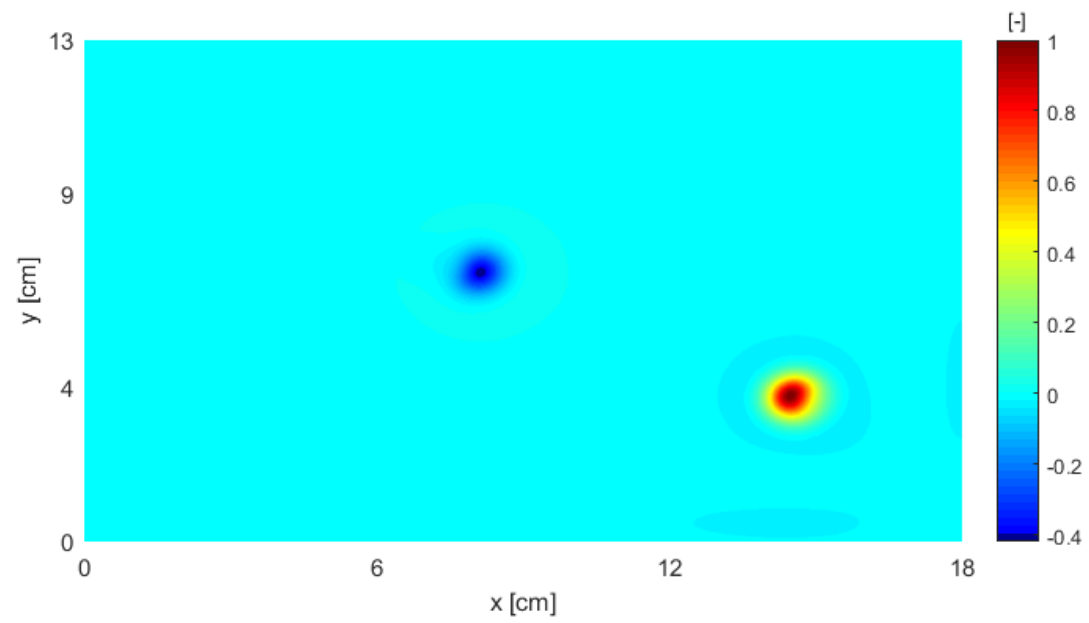

Figure A.4 - Normalized DSI field of the SI shown in Figure A.3. Since this map was extracted from a SI vector field that is heterogeneous, the concentrated positive- and negative-valued fields cannot be analyzed quantitatively. Its only purpose is to verify the location of the shaker and damper.

\section{References}

[1] D.U. Noiseux, Measurement of Power Flow in Uniform Beams and Plates, J. Acoust. Soc. Am. 47 (1970) 238. doi:10.1121/1.1911472.

[2] G. Pavić, Measurement of structure borne wave intensity, Part I: Formulation of the methods, J. Sound Vib. 49 (1976) 221-230. doi:10.1016/0022-460X(76)90498-3.

[3] R. Morikawa, S. Ueha, K. Nakamura, Error evaluation of the structural intensity measured with a scanning laser Doppler vibrometer and a k-space signal processing, J. Acoust. Soc. Am. 99 (1996) 2913-2921. doi:10.1121/1.414878.

[4] J.R.F. Arruda, P. Mas, Localizing Energy Sources and Sinks in Plates Using Power Flow Maps Computed From Laser Vibrometer Measurements, Shock Vib. 5 (1998) 235-253. doi:10.1155/1998/738387.

[5] N.B. Roozen, J.L. Guyader, C. Glorieux, Measurement-based determination of the irrotational part of the structural intensity by means of test functional series expansion, J. Sound Vib. 356 (2015) 168-180. doi:10.1016/j.jsv.2015.06.040.

390 [6] N.B. Roozen, J.L. Guyader, C. Glorieux, Using the irrotational part of the structural intensity to visualize vibrational energy sources, in: 23rd Int. Congr. Sound Vib., 2016: pp. 1-8.

[7] J.. Pascal, J.; Loyau, T. \& Mann III, Structural intensity from spatial Fourier transformation and BAHIM acoustical holography method, in: Proc. Cong. Struct. Intensity Vib. Energy Flow, Senlis, France, 1990: pp. 197-204.

[8] T. Eck, S.J. Walsh, Measurement of vibrational energy flow in a plate with high energy flow boundary crossing using electronic speckle pattern interferometry, Appl. Acoust. 73 (2012) 936- 
951. doi:10.1016/j.apacoust.2012.04.002.

[9] J.-C. Pascal, X. Carniel, V. Chalvidan, P. Smigielski, Determination of phase and magnitude of vibration for energy flow measurements in a plate using holographic interferometry, Opt. Lasers Eng. 25 (1996) 343-360. doi:10.1016/0143-8166(95)00080-1.

[10] M.J. Daley, S. a. Hambric, Simulating and measuring structural intensity fields in plates induced by spatially and temporally random excitation, J. Vib. Acoust. 127 (2005) 451-457. doi:10.1115/1.2980381.

[11] W.T. Schmidt, Open-crack Damage Assessments of Aluminum Panels Using Structural Intensitybased Techniques, The Pennsylvania State University, 2009. https://etda.libraries.psu.edu/paper/9843/4392.

[12] F. Semperlotti, S.C. Conlon, Structural damage identification in plates via nonlinear structural intensity maps., J. Acoust. Soc. Am. 127 (2010) EL48-53. doi:10.1121/1.3290175.

[13] C. Vuye, Measurement and modeling of sound and vibration fields using a scanning laser Doppler vibrometer, Vrije Universiteit Brussel, 2011.

[14] J.D. Blotter, R.L. West, S.D. Sommerfeldt, Spatially Continuous Power Flow Using a Scanning Laser Doppler Vibrometer, J. Vib. Acoust. 124 (2002) 476. doi:10.1115/1.1497363.

[15] L. Gavric, U. Carlsson, L. Feng, Measurement of structural intensity using a normal mode approach, J. Sound Vib. 206 (1997) 87-101. doi:10.1006/jsvi.1997.1077.

[16] H.G.D. Goyder, R.G. White, Vibrational power flow from machines into built-up structures, part I: Introduction and approximate analyses of beam and plate-like foundations, J. Sound Vib. 68 (1980) 59-75. doi:10.1016/0022-460X(80)90452-6.

[17] S.A. Hambric, Power flow and mechanical intensity calculations in structural finite element analysis, J. Vib. Acoust. 112 (1990) 542. doi:10.1115/1.2930140.

[18] L. Cremer, M. Heckl, B.A.T. Petersson, Structure-Borne Sound, Springer, Berlin \& Heidelberg, Germany, 2013. doi:10.1017/CBO9781107415324.004.

[19] J. Pascal, X. Carniel, J. Li, Characterization of a dissipative assembly by structural intensity using optical measurements, in: Fifth Int. Conf. Vib. Meas. by Laser Tech., 2002: pp. 180-191.

[20] N. Wang, C.; Ong, E.; Qian, H. \& Guo, On the application of B-spline approximation in structural intensity measurement, J. Sound Vib. 290 (2006) 508-513. doi:10.1016/j.jsv.2005.04.011.

[21] W.O. Wong, X.Q. Wang, L. Cheng, Modal power flow analysis of a damaged plate, J. Sound Vib. 320 (2009) 84-100. doi:10.1016/j.jsv.2008.07.004.

[22] G. Pavic, The role of damping on energy and power in vibrating systems, J. Sound Vib. 281 (2005) 45-71. doi:10.1016/j.jsv.2004.01.030.

430 [23] A. Lamberti, F. Semperlotti, Detecting closing delaminations in laminated composite plates using nonlinear structural intensity and time reversal mirrors, Smart Mater. Struct. 22 (2013) 125006. doi:10.1117/12.2009679.

[24] T. Jaber, M.; Schneeweiss, H.; Bös, J. \& Melz, Vibrational Energy Flow of Carbon Composite Structures materials and structures, in: Proc. Inter-Noise, Melbourne, Australia, 2014: p. 695.

[25] J.F. Arruda, Surface smoothing and partial spatial derivatives computation using a regressive discrete Fourier series, Mech. Syst. Signal Process. 6 (1992) 41-50. doi:10.1016/08883270(92)90055-N.

[26] R.G. Williams, E. G.; Dardy, H. D. \& Fink, A technique for measurement of structure-borne intensity in plates, J. Acoust. Soc. Am. 20375 (1985) 2061-2068. doi:10.1121/1.392663.

[27] J.-C. Pascal, J.-F. Li, X. Carniel, Wavenumber Processing Techniques to Determine Structural Intensity and Its Divergence from Optical Measurements without Leakage Effects, Shock Vib. 9 (2002) 57-66. doi:10.1155/2002/850152.

[28] J.-F. Li, J.-C. Pascal, C. Carles, A new K-space optimal filter for acoustic holography, in: Proc. 3rd Int. Congr. Air- Struct. Borne Sound Vib., Montreal, Canada, 1994: pp. 1-8. 
http://visualvibroacoustics.free.fr/fulltextpapers/1994CongressMontreal.pdf.

[29] M. Lopes, H.; Guedes, R. M. \& Vaz, Techniques in numerical differentiation of experimentally noisy data, in: 5th Int. Conf. Mech. Mater. Des., Porto, Portugal, 2006: pp. 27-28.

[30] T. Kreis, Handbook of Holographic Interferometry, Wiley-VCH Verlag GmbH \& Co. KGaA, Weinheim, Germany, 2004. doi:10.1002/3527604154.

450 [31] S. Jeught, J. Sijbers, J. Dirckx, Fast Fourier-Based Phase Unwrapping on the Graphics Processing Unit in Real-Time Imaging Applications, J. Imaging. 1 (2015) 31-44. doi:10.3390/jimaging1010031.

[32] D. De Greef, J. Soons, J.J.J. Dirckx, Digital Stroboscopic Holography Setup for Deformation Measurement at Both Quasi-Static and Acoustic Frequencies, Int. J. Optomechatronics. 8 (2014) 275-291. doi:10.1080/15599612.2014.942928.

[33] P. Guillaume, P. Verboven, S. Vanlanduit, H. Van der Auweraer, B. Peeters, A poly-reference implementation of the least- squares complex frequency-domain estimator, in: Proc. 21st Int. Modal Anal. Conf., Kissimmee, FL, USA, 2003: pp. 183-192.

[34] P. Heylen, W. and Lammens, S. and Sas, Modal analysis theory and testing, Katholieke Universiteit Leuven, Faculty of Engineering, Department of Mechanical Engineering, Division of Production Engineering, Machine Design and Automation, 1998. https://books.google.be/books?id=GS-IAAAACAAJ. 